\title{
Gauss Interpolation Formulas and Totally Positive Kernels*
}

\author{
By David L. Barrow
}

\begin{abstract}
This paper simplifies and generalizes an earlier result of the author's on Gauss interpolation formulas for the one-dimensional heat equation. Such formulas approximate a function at a point $\left(x^{*}, t^{*}\right)$ in terms of a linear combination of its values on an initial-boundary curve in the $(x, t)$ plane. The formulas are characterized by the requirement that they be exact for as many basis functions as possible. The basis functions are generated from a Tchebycheff system on the line $t=0$ by an integral kernel $K(x, y, t)$, in analogy with the way heat polynomials are generated from the monomials $x^{i}$ by the fundamental solution to the heat equation. The total positivity properties of $K(x, y, t)$ together with the theory of topological degree are used to establish the existence of the formulas.
\end{abstract}

1. Introduction. In a recent paper [1] we discussed formulas of the form

$$
u\left(x^{*}, t^{*}\right) \simeq \sum_{k=1}^{m} A_{k} u\left(x_{k}, t_{k}\right)
$$

for approximating solutions to the heat equation

$$
\frac{\partial u}{\partial t}=\frac{\partial^{2} u}{\partial x^{2}}
$$

The function $u$ is prescribed on an initial-boundary curve $C$ in the $(x, t)$ plane, and $\left(x^{*}, t^{*}\right)$ is a fixed point where an approximate solution is desired. The formula (1), where the points $\left(x_{k}, t_{k}\right)$ lie on $C$ and the weights $A_{k}$ are positive, is characterized by the requirement that it be exact for as many "basis functions" as possible. In [1] we proved the existence of $m$-point formulas which are exact for all heat polynomials of degree $n=2 m-1$, and that this is best possible, in the sense that no $m$-point formula is exact for all heat polynomials of degree $n_{1}>2 m-1$. Such formulas were called Gauss interpolation formulas, because of their similarity to Gaussian quadrature formulas.

A heat polynomial of degree $n$ is a linear combination of the functions

$$
u_{i}(x, t)=i ! \sum_{j=0}^{[i / 2]} \frac{x^{i-2 j} t^{j}}{(i-2 j) ! j !}, \quad i=0,1, \ldots, n,
$$

where $[a]$ means the greatest integer less than or equal to $a$. Each $u_{i}(x, t)$ solves (2) and satisfies

$$
u_{i}(x, 0)=x^{i} \equiv \phi_{i}(x) .
$$

If we now introduce the fundamental solution to the heat equation

Received July $1,1976$.

AMS (MOS) subject classifications (1970). Primary 65N99; Secondary 65D05.

*This work was supported by NSF Grant DC R75-04545. 


$$
K(x, y, t)=\frac{1}{\sqrt{4 \pi t}} \exp \left(-(x-y)^{2} / 4 t\right), \quad t>0
$$

we can express the heat polynomials as

$$
u_{i}(x, t)=\int_{-\infty}^{\infty} K(x, y, t) \phi_{i}(y) d y, \quad t>0
$$

Now, it is known that for each $t>0$, the kernel $K$ is extended totally positive (cf. Karlin [2], and below), and that the functions $\phi_{i}(x)=x^{i}, i=0,1, \ldots, n$, form a Tchebycheff system (cf. Karlin and Studden [3]). These facts can be used to simplify the proof of the result from [1] mentioned above. More significantly, the concept of total positivity allows considerable generalization of the results of [1]. For example, Karlin and McGregor have shown that fundamental solutions for a large class of one-dimensional parabolic problems are totally positive [5]. If such fundamental solutions can be shown to satisfy a little more, namely, the hypotheses of Theorem 1 below (as in fact some of the specific examples from [4] seem to do), then we will have shown the existence of Gauss interpolation formulas for solutions to these parabolic problems. In Section 3 we prove that one of the examples from [4] does indeed satisfy the hypotheses of Theorem 1; we also give a numerical example based on this case.

A further generalization incorporated into Theorem 1 is the consideration of formulas of the form

$$
u\left(x^{*}, t^{*}\right) \simeq \sum_{k=1}^{m} A_{k}\left(u+\alpha \frac{\partial u}{\partial x}\right)\left(x_{k}, t_{k}\right),
$$

where $\alpha=\alpha(x, t)$ is a prescribed continuous function on the curve $C$. Such a formula could be used, for example, in the case where the data $a u+b u_{x}=f$ is known on $C$, for $a$ and $b$ fixed continuous functions with $a>0$. One first obtains the formula (4) corresponding to $\alpha=b / a$, and then applies it to the data $f / a$.

In the next section we state and prove the main results of this paper, Theorems 1 and 2 . Theorem 2 is concerned with the linear independence of certain linear functionals on a space of functions of two variables, and can be thought of as providing a "zero-counting" procedure for such functions. The corollary to Theorem 2 is a key step in the proof of Theorem 1.

2. Main Results. We first introduce some notation for certain determinants associated with a function $K(x, y)$ (cf. Karlin [2, Chapter 2]). Here $x$ and $y$ take values in the totally ordered sets $X$ and $Y$, respectively. Let $x_{1}<x_{2}<\cdots<x_{r}$ be selections from $X$, and $y_{1}<y_{2}<\cdots<y_{r}$ from $Y$. The determinant of the matrix whose $(i, j)$ th entry is $K\left(x_{i}, y_{j}\right)$ will be denoted by

$$
K\left(\begin{array}{l}
x_{1}, x_{2}, \ldots, x_{r} \\
y_{1}, y_{2}, \ldots, y_{r}
\end{array}\right) \text {. }
$$

If this determinant is nonnegative for all such choices of the $x_{i}$ 's and $y_{j}$ 's, then $K$ is said to be totally positive.

If $X$ is an open interval and $x_{1} \leqslant x_{2} \leqslant \cdots \leqslant x_{r}$ are values from $X$ and $y_{1}<y_{2}$ $<\cdots<y_{r}$, then 


$$
K^{*}\left(\begin{array}{l}
x_{1}, x_{2}, \ldots, x_{r} \\
y_{1}, y_{2}, \ldots, y_{r}
\end{array}\right)
$$

will denote the determinant of the matrix described as follows: if $x_{i}=x_{i+1}=\cdots=$ $x_{i+l}$ is a block of coincident $x$ 's, then the $(i+k)$ th row of the matrix will have the entries

$$
\frac{\partial^{k}}{\partial x^{k}} K\left(x_{i}, y_{j}\right), \quad k=0,1, \ldots, l
$$

(assuming $K$ is sufficiently differentiable). If this determinant is always positive whenever at most $r$ of the $x$ 's coincide, we say that $K$ is extended totally positive of degree $r$ in the variable $x$.

We now state the hypotheses for Theorem 1 . Let $K(x, y, t)$ be a real valued function continuous on $X \times X \times(0, \infty)$, where $X$ is an open interval. $K$ is assumed to have the following properties:

$\mathrm{K} 1$. For each $t>0, K$ is extended totally positive of degree 3 in the variable $x$;

K2. There is a class of functions $D_{K}$ which are integrable with respect to the measure $d \mu=K(x, y, t) d y$ over the interval $X$; furthermore, we assume that if $f \in D_{K}$, and if

$$
\begin{aligned}
T_{t} f(x) & \equiv \int_{X} K(x, y, t) f(y) d y, \quad t>0, \\
& \equiv f(x), \quad t=0
\end{aligned}
$$

then $T_{t} f(x)$ is continuous for $(x, t) \in X \times[0, \infty)$ if $f \in C(X)$; also, we assume that differentiation under the integral sign up to order 2 in $x$ is legitimate:

$$
\frac{\partial^{\nu} T_{t} f(x)}{\partial x^{\nu}}=\int_{X} \frac{\partial^{\nu} K(x, y, t)}{\partial x^{\nu}} f(y) d y, \quad \nu=0,1,2, t>0
$$

K3. $\int_{X} K(x, y, t) d y=1$ for $t>0$.

K4. The family of linear operators $T_{t}$ is a semigroup: $T_{s+t}=T_{t} \circ T_{s}$.

Now let $n$ be a positive integer and let $\left\{\phi_{i}\right\}_{i=0}^{n} \subset C^{2}(X) \cap D_{K}$ be an extended Tchebycheff system of order 3 (cf. [3, p. 6]). This is equivalent modulo the sign of one of the functions $\phi_{i}$, to the statement that any polynomial $p(x)=\Sigma_{i=0}^{n} \beta_{i} \phi_{i}(x)$ has at most $n$ zeroes, counting multiplicities up to order 3 . Also, we specify that $\phi_{0}(x) \equiv 1$.

We next define the family of functions

$$
u_{i}(x, t) \equiv T_{t} \phi_{i}(x), \quad i=0,1, \ldots, n,
$$

in analogy with (3). We can now state

THEOREM 1. Let $C:\{(x(s), t(s)): 0 \leqslant s \leqslant 1\}$ be a Jordan arc in the $(x, t)$ plane satisfying:

(i) $x(s) \in X, 0 \leqslant s \leqslant 1$;

(ii) $(x(0), t(0))=\left(a, t^{*}\right),(x(1), t(1))=\left(b, t^{*}\right)$, with $a<b$ and $t^{*}>0$;

(iii) $0 \leqslant t(s)<t^{*}$ for $0<s<1$.

Let $\alpha(s)$ be continuous for $0 \leqslant s \leqslant 1$, and consider $\alpha$ to be defined on $C$ via the parametrization for $C: \alpha(x(s), t(s))=\alpha(s)$. Assume $\alpha\left(a, t^{*}\right) \leqslant 0 \leqslant \alpha\left(b, t^{*}\right)$. Then for 
any $a<x^{*}<b$ and $n=2 m-1(m \geqslant 2)$, there is a formula of the form

$$
u\left(x^{*}, t^{*}\right) \simeq \sum_{k=1}^{m} A_{k}\left(u+\alpha \frac{\partial u}{\partial x}\right)\left(x_{k}, t_{k}\right)
$$

which is exact for all polynomials $p(x, t)=\Sigma_{i=0}^{n} \beta_{i} u_{i}(x, t)$. The weights $A_{k}$ are positive and the points $\left(x_{k}, t_{k}\right)$ lie on $C$, with $t_{k}<t^{*}$. Furthermore, no such formula can hold for all polynomials of degree $n_{1}>n$.

The proof of Theorem 1 will be postponed until we establish some lemmas and Theorem 2.

Lemma 1. For $f \in C^{2}(X)$, let $\widetilde{Z}_{(3)}(f)$ be the number of zeroes of $f$, counting multiplicities up to order 3 , and let $S(f)$ be the number of strict sign changes of $f$ on $X$. Then for $f \in C^{2}(X) \cap D_{K}, f \neq 0$, and $t>0$,

$$
\widetilde{Z}_{(3)}\left(T_{t} f\right) \leqslant S(f) \text {. }
$$

Proof. The proof is identical to the proof of the (a) part of Theorem 3.2, p. 239 of [2]. The requirement there that $f$ be bounded is obviated by our hypotheses on $K$ and $D_{K}$.

Corollary. If $f \in D_{K}, f \neq 0$ and $f \geqslant 0$, then $T_{t} f(x)>0$ for $t>0$ and $x \in X$.

Proof. $S(f)=0$, so $T_{t} f$ has no zeroes, by the lemma. Hence, $T_{t} f$ is positive (it is nonnegative since $K(x, y, t)$ is, as follows from its total positivity).

For convenience, we introduce the vector notation

$$
\mathbf{u}(x, t)=\left(u_{0}(x, t), u_{1}(x, t), \ldots, u_{n}(x, t)\right)
$$

and

$$
\phi(x)=\left(\phi_{0}(x), \phi_{1}(x), \ldots, \phi_{n}(x)\right)=\mathbf{u}(x, 0)
$$

THEOREM 2. Let the functions $\left\{u_{i}(x, t)\right\}, i=0,1, \ldots, n$, be as in the hypotheses for Theorem 1. Let $\left(x_{k}, t_{k}\right), k=1,2, \ldots, l+j, l \geqslant 0, j \geqslant 1$, be distinct points in the half-plane $t \geqslant 0$ such the the first $l$ of them have equal $t$-coordinates which are greater than or equal to those of the other points; i.e., $t_{1}=t_{2}=\cdots=$ $t_{l} \geqslant t_{k}, k=l+1, \ldots, l+j$. Suppose also that $n+1 \geqslant l+2 j$. Then the vectors

$$
\left\{\mathbf{u}\left(x_{k}, t_{k}\right)\right\}_{k=1}^{l+j} \cup\left\{\frac{\partial \mathrm{u}}{\partial x}\left(x_{k}, t_{k}\right)\right\}_{k=l+1}^{l+j}
$$

are linearly independent.

Proof. We may assume that $n+1=l+2 j$, for otherwise we could adjoin points to the line $t=t_{1}$, increasing $l$, to achieve this. Suppose the theorem were false. Then there would exist a nontrivial polynomial $p(x, t)=\sum_{i=0}^{n} \beta_{i} u_{i}(x, t)$ satisfying

$$
p\left(x_{k}, t_{k}\right)=0, \quad k=1,2, \ldots, l+j,
$$

and

$$
P_{x}\left(x_{k}, t_{k}\right)=0, \quad k=l+1, \ldots, l+j .
$$

We will show that this is impossible.

For $t \geqslant 0$, let $\widetilde{Z}(t)$ denote the number of zeroes of $p(x, t)$ in $x$, counting 
multiplicities up to order three. Let $S(t)$ be the number of sign changes of $p(x, t)$ in $x$. Lastly, let $j=j_{1}+j_{2}$, where $j_{1}$ is the number of the points $\left(x_{k}, t_{k}\right), k=l+1, \ldots$, $l+j$, on the line $t=t_{1}$. Then clearly

$$
\widetilde{Z}\left(t_{1}\right) \geqslant l+2 j_{1}
$$

and

$$
\widetilde{Z}(0) \leqslant n,
$$

the last inequality holding because the functions $u_{i}(x, 0)$ form an extended Tchebycheff system of order 3.

It will now be convenient to classify the types of zeroes that $p(x, t)$ may have.

For $t \geqslant 0$, let $z_{i}(t), i=1,2,3$, denote the number of $x$ 's such that:

for $i=1, p(x, t)=0, p_{x}(x, t) \neq 0$;

for $i=2, p(x, t)=p_{x}(x, t)=0, p$ does not change sign at $x$;

for $i=3, p(x, t)=p_{x}(x, t)=0, p$ does change sign at $x$.

Thus, each $\left(x_{k}, t_{k}\right)$ with $k=l+1, \ldots, l+j$ is a zero of type $z_{2}$ or $z_{3}$. Furthermore, it is clear that

$$
\widetilde{Z}(t) \geqslant z_{1}(t)+2 z_{2}(t)+3 z_{3}(t) \geqslant z_{1}(t)+z_{3}(t)=S(t) .
$$

By Lemma 1 , we have for any $\delta>0$,

$$
\widetilde{Z}(t+\delta)=\widetilde{Z}_{(3)}\left(T_{\delta} p(\cdot, t)\right) \leqslant S(p(\cdot, t))=S(t) .
$$

Combining (10) and (11), we obtain

$$
\widetilde{Z}(t+\delta) \leqslant S(t)=z_{1}(t)+z_{3}(t) \leqslant \widetilde{Z}(t) \geqslant z_{1}(t)+2 z_{2}(t)+3 z_{3}(t) .
$$

Hence, $\widetilde{Z}(t)$ is nonincreasing in $t$ and decreases by at least two due to each of the points $\left(x_{k}, t_{k}\right), k=l+1, \ldots, l+j$, where $p=p_{x}=0$. Thus we have

$$
\widetilde{Z}\left(t_{1}\right) \leqslant \widetilde{Z}(0)-2 j_{2} \leqslant n-2 j_{2}=l+2 j_{1}-1,
$$

which is a contradiction of (8).

COROLlary. Assuming all the hypotheses of Theorem 1, a necessary condition for the existence of a formula (7) with $A_{k} \geqslant 0$ and $\left(x_{k}, t_{k}\right) \in C$ which is exact for polynomials of degree $n=2 m-1$ or less, is that all $m$ points be distinct, and for $k=1,2, \ldots, m, A_{k}>0$ and $t_{k}<t^{*}$.

Proof. We first show that a formula with fewer than $m$ points is impossible. Suppose that

$$
\mathbf{u}\left(x^{*}, t^{*}\right)=\sum_{k=1}^{j} A_{k}\left(\mathrm{u}+\alpha \mathbf{u}_{x}\right)\left(x_{k}, t_{k}\right),
$$

where $j<m$. If we now adjoin points to the line $t=t^{*}$, to give $l=2(m-j)$ in all, and reindex, we see that (14) is impossible by Theorem 2 .

To see that $t_{k}<t^{*}$ for all $k$, suppose first that $t_{1}=t^{*}$ and $t_{k}<t^{*}, k=2$, $\ldots, m$. If we take $l=0$ and $j=m$, Theorem 2 implies the existence of a polynomial $p(x, t)=\sum_{i=0}^{n} \beta_{i} u_{i}(x, t)$ satisfying $p\left(x_{k}, t_{k}\right)=0, k=1, \ldots, m ; p_{x}\left(x_{k}, t_{k}\right)=0, k=$ $2, \ldots, m$; and $p_{x}\left(x_{1}, t_{1}\right)=1$. But then the formula (7) gives 


$$
p\left(x^{*}, t^{*}\right)=\sum_{k=1}^{m} A_{k}\left(p+\alpha p_{x}\right)\left(x_{k}, t_{k}\right)=A_{1} \alpha\left(a, t^{*}\right) \leqslant 0 .
$$

This in turn implies that $p\left(x, t^{*}\right)$ has at least two distinct zeroes in $x$, which, by Theorem 2 with $l=2$ and $j=m-1$, implies $p \equiv 0$, a contradiction. The other two possibilities, $t_{1}<t_{m}=t^{*}$ and $t_{1}=t_{m}=t^{*}$ are dispensed with similarly. This completes the proof of the corollary.

Lемма 2. The vector $q \equiv \mathrm{u}\left(x^{*}, t^{*}\right)$ has a unique representation of the form

$$
q=\sum_{k=1}^{m} \lambda_{k} \phi\left(x_{k}\right)
$$

where $x_{k} \in X$ and $\lambda_{k}>0, k=1, \ldots, m$.

Proof. Since $u_{i}\left(x^{*}, t^{*}\right)=\int_{X} K\left(x^{*}, y, t^{*}\right) \phi_{i}(y) d y, i=0,1, \ldots, n$, the components of $q$ form a "moment sequence" with respect to the functions $\left\{\phi_{i}(x)\right\}$ on $X$. It follows by Theorem 1 of [7] that there is a representation for $q$ of the form

$$
q=\sum_{k=1}^{p} \gamma_{k} \phi\left(y_{k}\right)
$$

with $\gamma_{k}>0, y_{k} \in X, k=1, \ldots, p$. Let $c, d$ be such that $\left\{y_{k}\right\} \subset(c, d) \subset X$. Since the functions $\left\{\phi_{i}\right\}, i=0, \ldots, n$, form a Tchebycheff system on $[c, d]$, we may appeal to the results of [3, Chapter 2]. The equation (16) shows that $q$ belongs to the "moment cone" generated by the $\left\{\phi_{i}\right\}$ on $[c, d]$ (i.e., those vectors c whose components $c_{i}=\int_{c}^{d} \phi_{i}(s) d \mu(s)$ for some bounded, right-continuous function $\left.\mu(s)\right)$. Moreover, the Corollary to Lemma 1 implies that $q$ is actually in the interior of the moment cone. The conclusion of Lemma 2 now follows from Corollary 3.1, p. 47 of [3].

Proof of Theorem 1. As in [1], the proof uses the concept of topological degree to establish the existence of a solution to a system of $N$ equations in $N$ unknowns. We begin by reviewing the needed properties of degree theory (see Schwartz [8] or Ortega and Rheinboldt [6]).

Let $D$ be an open bounded set in the Euclidean space $R^{N}$, with $\bar{D}$ and $\partial D$ denoting its closure and boundary, respectively. Let $F: \bar{D} \rightarrow R^{N}$ be continuous. Then if $q \in R^{N}$ and $q \notin F(\partial D)$, the degree of $F$ with respect to $D$ and $q$ is defined, has an integer value, and will be denoted by $\operatorname{deg}(F, D, q)$. The following are some basic properties of the degree:

(i) Suppose that $F \in C^{\prime}(D), q \notin F(\partial D)$, and that for each $z \in D$ where $F(z)=q$, it is true that $\operatorname{det}\left(F^{\prime}(z)\right) \neq 0$. Then there are a finite number of points $z_{i} \in D$ where $F\left(z_{i}\right)=q$, and $\operatorname{deg}(F, D, q)=\Sigma_{i} \operatorname{sgn}\left(\operatorname{det}\left(F^{\prime}\left(z_{i}\right)\right)\right)$.

(ii) If $\operatorname{deg}(F, D, q) \neq 0$, there is at least one point $z \in D$ such that $F(z)=q$.

(iii) Let $F(z, \lambda)$ be continuous on $\bar{D} .[0,2]$, such that $F(z, \lambda) \neq q$ for any $z \in \partial D, 0 \leqslant \lambda \leqslant 2$. Then $\operatorname{deg}(F(\cdot, \lambda), D, q)$ is constant, independent of $\lambda$.

We will apply these properties by constructing a function $F(z, \lambda)$, a set $D$, and a point $q$, such that $\operatorname{deg}(F(\cdot, 0), D, q)= \pm 1, F(z, \lambda) \neq q$ for $z \in \partial D, 0 \leqslant \lambda \leqslant 2$, and hence deduce that $\operatorname{deg}(F(\cdot, 2), D, q)= \pm 1$. This will imply that the equation $F(z, 2)$ $=q$ has a solution in $D$, which will be equivalent to the existence statement of Theorem 1 . 
Let $N=2 m=n+1$ and let $D \subset R^{N}$ be the set

$$
\begin{aligned}
D=\{z= & \left(A_{1}, A_{2}, \ldots, A_{m}, s_{1}, s_{2}, \ldots, s_{m}\right): \\
& \left.0<s_{1}<s_{2}<\cdots<s_{m}<1,0<A_{k}<1, k=1, \ldots, m\right\} .
\end{aligned}
$$

Let $\mathbf{u}(x, t)$ be as before, and let $q=\mathrm{u}\left(x^{*}, t^{*}\right)$. Let $C_{0}:\left\{\left(x_{0}(s), t_{0}(s)\right), 0 \leqslant s \leqslant 1\right\}$ be a Jordan arc to be described below, and let $C_{1}$ be the curve $C$ of Theorem 1 (parametrized by $\left.x_{1}(s) \equiv x(s), t_{1}(s) \equiv t(s)\right)$. For $(s, \lambda) \in[0,1] \times[0,1]$, let $C_{\lambda}$ be the curve

$$
(x(s, \lambda), t(s, \lambda))=\left(\lambda x_{1}(s)+(1-\lambda) x_{0}(s), \lambda t_{1}(s)+(1-\lambda) t_{0}(s)\right) .
$$

Thus, as $\lambda$ varies from 0 to $1, C_{\lambda}$ is a continuous deformation of $C_{0}$ into $C_{1}$.

We define $F(z, \lambda)$ as

$$
\begin{aligned}
F(z, \lambda) & =\sum_{k=1}^{m} A_{k} \mathbf{u}\left(x\left(s_{k}, \lambda\right), t\left(s_{k}, \lambda\right)\right), \quad 0 \leqslant \lambda \leqslant 1, \\
& =\sum_{k=1}^{m} A_{k}\left(\mathbf{u}+(\lambda-1) \alpha \mathbf{u}_{x}\right)\left(x_{1}\left(s_{k}\right), t_{1}\left(s_{k}\right)\right), \quad 1<\lambda \leqslant 2 .
\end{aligned}
$$

Let $C_{0}:\left(x_{0}(s), t_{0}(s)\right)$ be any Jordan arc (i.e., continuous, non-self-intersecting) satisfying:

(a) $\left(x_{0}(0), t_{0}(0)\right)=\left(a, t^{*}\right),\left(x_{0}(1), t_{0}(1)\right)=\left(b, t^{*}\right)$

(b) $\left(x_{0}(s), t_{0}(s)\right) \subset X \times\left[0, t^{*}\right)$ for $0<s<1$;

(c) $C_{0}$ includes an open interval of the $x$-axis which contains the points $\left\{x_{k}\right\}$ of Lemma 2, with the parametrization chosen so that if $x_{k}=x_{0}\left(s_{k}\right)$, then $d x_{0}\left(s_{k}\right) / d s=1$.

Lemma 3. If $z \in \partial D$ and $0 \leqslant \lambda \leqslant 2$, then $F(z, \lambda) \neq q$.

Proof. This is an immediate application of the Corollary to Theorem 2. Note that if $z \in \partial D$, one or more of the following is true:

(i) $s_{1}=0$ or $s_{m}=1$,

(ii) $s_{k}=s_{k+1}$ for some $k=1,2, \ldots, m-1$,

(iii) some $A_{k}=0$,

(iv) some $A_{k}=1$.

Thus, if $z \in \partial D$ and $F(z, \lambda)=q$ with one of the first three cases occurring, there would exist a formula (7) of a kind ruled out by the corollary (applied with a possibly different curve $C$ or function $\alpha$ ). In case (iv), we use the fact that $u_{0}(x, t) \equiv 1$ so that $\sum_{i=1}^{m} A_{i}=1$, and conclude that case (iii) must also hold.

We now claim that

$$
\operatorname{deg}(F(\cdot, 0), D q)= \pm 1 \text {. }
$$

The fact that $F(z, 0)=q$ has a unique solution in $D$ follows immediately from Lemma 2. At this solution,

$$
\frac{\partial F(z, 0)}{\partial z}=\left[\phi\left(x_{1}\right), \ldots, \phi\left(x_{m}\right), A_{1} \frac{d \phi}{d x}\left(x_{1}\right), \ldots, A_{m} \frac{d \phi}{d x}\left(x_{m}\right)\right],
$$

where $x_{1}, x_{2}, \ldots, x_{m}$ are the points of Lemma 2. The determinant of this matrix is 
nonzero, since the positive $A_{k}$ 's may be factored out and the $\left\{\phi_{i}\right\}, i=0, \ldots, n$, form an extended Tchebycheff system of order three. The equality (18) now follows from property (i) of the degree.

Combining (18) and Lemma 3 with properties (ii) and (iii) of the degree, we deduce that the equation $F(z, 2)=q$ has a solution $z \in D$. This proves the existence statement of Theorem 1. The fact that such a formula cannot hold for all polynomials of degree $n_{1}>n$ follows from Theorem 2 .

3. An Example. We conclude with an example illustrating an instance of Theorem 1. It should be pointed out that the example is not arbitrary, but was chosen because the fundamental solution $K(x, y, t)$ and the family of solutions $\left\{u_{i}(x, t)\right\}$ used in Theorem 1 are known explicitly for this case.

Consider the diffusion equation [4, pp. 170-171] on $X=(-\infty, \infty)$

$$
\frac{\partial u}{\partial t}=e^{x^{2}} \frac{\partial}{\partial x}\left(e^{-x^{2}} \frac{\partial u}{\partial x}\right)=\frac{\partial^{2} u}{\partial x^{2}}-2 x \frac{\partial u}{\partial x}
$$

The Cauchy problem for this equation has the fundamental solution

$$
K(x, y, t)=a(t) \exp \left(-b(t) x^{2}\right) \exp \left(-b(t) y^{2}\right) \exp (c(t) x y)
$$

where $a(t)=\left(\pi\left(1-e^{-4 t}\right)\right)^{-1 / 2}, b(t)=e^{-4 t} /\left(1-e^{-4 t}\right)$, and $c(t)=2 e^{-2 t} /\left(1-e^{-4 t}\right)$. We first show that, for each $t>0$, this kernel is extended totally positive, of arbitrary degree, in both $x$ and $y$. Indeed, by Theorem 2.6, p. 55 of [2], it is sufficient to show that for $m=1,2, \ldots$,

$$
K^{*}(\overbrace{x, \ldots, x}^{m} \begin{array}{c}
m, \ldots, y
\end{array})>0
$$

(where we suppress the dependence of $K$ on $t$ ); $(21)$ is established by arguing as in [2, pp. 99-100], where (21) is proved for $K(x, y)=e^{x y}$. Properties K2-K4 likewise can be shown to hold.

We take for the functions $\left\{u_{i}(x, t)\right\}$ the class of solutions to (19)

$$
u_{i}(x, t)=e^{-2 i t} H_{i}(x), \quad i=0,1, \ldots,
$$

where $H_{i}(x)=(-1)^{i} e^{x^{2}} d^{i} / d x^{i} e^{-x^{2}}$ are the Hermite polynomials. We let the coefficient $\alpha$ in (7) be zero and take for the image of the curve $C$ the set $(0, t): 0 \leqslant t \leqslant .1,(x, 0)$ : $0 \leqslant x \leqslant 1$, and $(1, t), 0 \leqslant t \leqslant .1$. Formulas (7) were calculated for $t^{*}=.1$ and $x^{*}=$ $.25, .5$, and .75 , with $m=2,3, \ldots, 6$, by numerically solving $2 m$ nonlinear equations in each case.

The formulas for $\left(x^{*}, t^{*}\right)=(.5, .1)$ are given in Table 1 . Table 2 presents the result of applying these formulas to the problem of interpolating the function $u(x, t)$ which satisfies (19) and

this function being $u(x, t)=e^{2 t+x^{2}}$.

$$
\begin{aligned}
& u(0, \mathrm{t})=e^{2 t}, \\
& u(x, 0)=e^{x^{2}}, \quad 0 \leqslant x \leqslant 1, \\
& u(1, t)=e^{2 t+1},
\end{aligned}
$$




\section{TABLE 1}

Interpolation formulas for $\left(x^{*}, t^{*}\right)=(.5, .1)$

\begin{tabular}{|c|c|c|c|}
\hline m & $\mathrm{A}_{\mathrm{k}}$ & $x_{k}$ & $t_{k}$ \\
\hline 2 & $\begin{array}{l}.5 \\
.5\end{array}$ & $\begin{array}{l}.33604788(-2) \\
.81537027\end{array}$ & $\begin{array}{l}.0 \\
.0\end{array}$ \\
\hline 3 & $\begin{array}{l}.35269431 \\
.41343840 \\
.23386729\end{array}$ & $\begin{array}{l}.0 \\
.45103057 \\
1.0\end{array}$ & $\begin{array}{l}.38492690(-1) \\
.0 \\
.24033195(-1)\end{array}$ \\
\hline 4 & $\begin{array}{l}.26874349 \\
.26561142 \\
.27676509 \\
.18888000\end{array}$ & $\begin{array}{l}.0 \\
.19419862 \\
.66696976 \\
1.0\end{array}$ & $\begin{array}{l}.52478250(-1) \\
.0 \\
.0 \\
.43361146\end{array}$ \\
\hline 5 & $\begin{array}{l}.20936116 \\
.15094470 \\
.35320546 \\
.13407306 \\
.15241562\end{array}$ & $\begin{array}{l}.0 \\
.0 \\
.43742786 \\
.87996367 \\
1.0\end{array}$ & $\begin{array}{l}.60423741(-1) \\
.28924570(-2) \\
.0 \\
.0 \\
.53738453(-1)\end{array}$ \\
\hline 6 & $\begin{array}{l}.15282969 \\
.16747343 \\
.22581758 \\
.24931337 \\
.87309498 \quad(-1) \\
.11725642\end{array}$ & $\begin{array}{l}.0 \\
.0 \\
.27120875 \\
.63648509 \\
1.0 \\
1.0\end{array}$ & $\begin{array}{l}.67010371(-1) \\
.20535510(-1) \\
.0 \\
.0 \\
.88217190(-2) \\
.61648245(-1)\end{array}$ \\
\hline
\end{tabular}

TABLE 2

Interpolation formulas applied to $u(x, t)=\exp \left(2 t+x^{2}\right)$

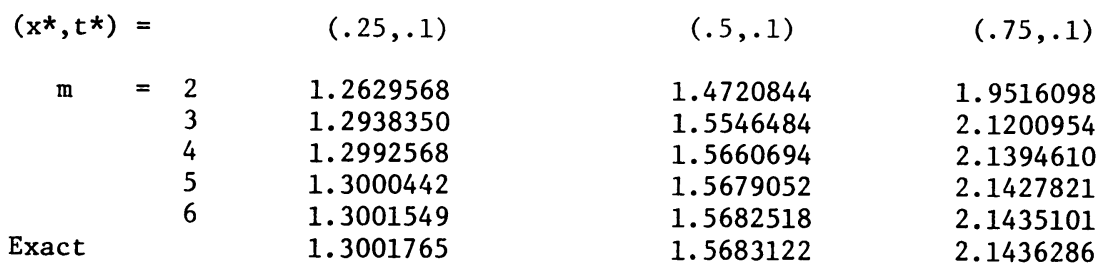

All calculations described in this paper were performed on the AMDAHL 470 computer at the Data Processing Center at Texas A \& M University, using double precision arithmetic in FORTRAN, which carries approximately 16 significant digits.

Department of Mathematics

Texas A \& M University

College Station, Texas 77801

1. D. L. BARROW, "Existence of Gauss interpolation formulas for the one-dimensional heat equation," Math. Comp., v. 30, 1976, pp. 24-34.

2. S. KARLIN, Total Positivity. Vol. I, Stanford Univ. Press, Stanford, California, 1968. MR 37 \#5667. 
3. S. KARLIN \& W. J. STUDDEN, Tchebycheff Systems: With applications in Analysis and Statistics, Interscience, New York, 1966. MR 34 \#757.

4. S. KARLIN \& J. MCGREGOR, "Classical diffusion processes and total positivity," $J$. Math. Anal. Appl., v. 1, 1960, pp. 163-183. MR 22 \#12574.

5. S. KARLIN \& J. McGREGOR, "Total positivity of fundamental solutions of parabolic equations," Proc. Amer. Math. Soc., v. 13, 1962, pp. 136-139. MR 24 \#A2761.

6. J. M. ORTEGA \& W. C. RHEINBOLDT, Iterative Solution of Nonlinear Equations in Several Variables, Academic Press, New York and London, 1970. MR 42 \#8686.

7. W. W. ROGOSINSKI, "Moments of non-negative mass," Proc. Roy. Soc. London, Ser. $A$, v. 245, 1958, pp. 1-27. MR $26 \# 6709$.

8. J. T. SCHWARTZ, Nonlinear Functional Analysis, Gordon and Breach, New York, 1969. 